\title{
Histo-epidemiological aspect of Helicobacter pylori chronic gastritis in a Moroccan population
}

\author{
Radia El Guil ${ }^{1,}$, Hajar Hechlaf $^{2}$, Soumia Ed-Day ${ }^{1}$, Omar Akhouayri $^{1}$, Samira Boulbaroud $^{3}$, and Fatima-Zahra Azzaoui ${ }^{1}$ \\ ${ }^{1}$. Laboratory of Biology and Health, Faculty of Sciences, Kenitra, Morocco. \\ 2. Pathological anatomy and cytology laboratory, El Idrissi hospital, Kenitra, Morocco \\ ${ }^{3}$. Polydisciplinary Faculty, Beni Mellal, Morocco.
}

\begin{abstract}
Helicobacter pylori (H. Pylori) is the best example of the implication of chronical infection in carcinogenesis. The World Health Organization recognized it as a class I carcinogen since it triggers the progression of premalignant gastric lesions. The aim of this study is to define the prevalence of $H$. Pylori infection, related risk factors, and explore the histological features of the chronic gastritis. This is a retrospective study of 248 gastric specimens, examinated and evaluated according Sydney to system. The prevalence of $H$. Pylori is $67 \%$, chronic gastritis is observed in all the biopsies (100\%). Age and gender were not a risk factors for the $\mathrm{H}$. Pylori infection. Prevalence of $H$. Pylori was $71.93 \%$ in low socio-economic class. The severity of the chronic gastritis increases if the colonization of $H$. Pylori increases. In $74,58 \%$ of cases chronic gastritis was active. Glandular atrophy presented $10.37 \%$ and in $80 \%$ was related to $H$. Pylori infection $(p=0,004)$ and $12 \%$ related to autoimmune diseases. The prevalence of Intestinal metaplasia is $10.53 \%$, and $H$. Pylori was observed in $42.31 \%$ of cases $(p=0.001)$. The dysplasia is detected in one case, in a 72 years old patient. We also observed one case of gastric adenocarcinoma, of an 80 years. Follicular gastritis are in $32 \%$ of cases, and they were more frequent in $\mathrm{H}$. Pylori infected slides $(82 \%$ of subjects) ( $p<0.005)$.According to this study, $H$. Pylori Chronic Gastritis is very common in our population, and a coherent relationship exists between $\mathrm{H}$. Pylori colonization and pre-cancerous lesions. An early eradicating should be considered as a health goal.
\end{abstract}

Keywords. Helicobacter Pylori, Chronic gastritis, Precancerous lesions, Epidemiology, Morocco.

\section{Introduction}

H. Pylori is the best example of the implication of chronic infection in carcinogenesis, we estimate that approximately $50 \%$ of the word population is affected by this bacteria [1], While the infection is usually occurs in childhood, there is typically a long period of latency with disease manifestations not appearing until adulthood. Gastric cancer does not usually manifest until old age [2]. A significant differences in prevalence exist both within and between countries. Generally, the overall prevalence is higher in countries of underdeveloped regions, such as Africa and Asia, than in the more developed countries like Western Europe and North America [3]. It has now been well established that infection of the human host with H. Pylori is an important factor in the etiology of gastric-related diseases [4] ranging from active chronical gastritis to gastric cancer. The World Health Organization (WHO) recognized H. Pylori as a class I carcinogen, and estimates that gastric cancer will be the 11th leading cause of mortality by 2030 [5].
Correa proposed a human model of gastric cancer based on histological precancerous lesions in the 1975 [6], in fact gastric cancer is the end result of progressive evolution of changes in the gastric mucosa, starting with chronic gastritis, followed by atrophic gastritis then intestinal metaplasia, dysplasia and finally the point of no return, the invasive adenocarcinoma.

The present study was undertaken to define the prevalence of $H$. Pylori infection, on 248 subject suffering from different gastric pathology that required an endoscopy, studying the effect of different epidemiological factors such as, age, gender, socioeconomic status, and explore the histological features of chronic gastritis, with special emphasis on the presence of premalignant changes of the gastric mucosa, such as IM, atrophy, and dysplasia presence or absence of H. Pylori, activity (neutrophilic infiltration) of gastric mucosa and presence or absence of lymphoid aggregates.

\section{Materials and Methods}

${ }^{*}$ Corresponding author: radia.elgui@uit.ac.ma 


\subsection{Study identification and extraction of data}

It's a retrospective descriptive study performed on all gastric biopsies received in the pathological anatomy and cytology laboratory of EL Idrissi Public Hospital, Kenitra, Morocco, between March 2017 and March 2019. 248 specimens were collected, belonging to patients who had Esophagogastroduodenoscopy (EGD) due to different gastric disorders.

Data including age, gender, indication for EGD, and pathology reports were acquired from the laboratory medical records.

\subsection{Histopathology}

Biopsy specimens, fixed in $10 \%$ buffered formaldehyde solution, embedded in paraffin, and cut in sequential $4 \mu \mathrm{m}$ sections, and stained with hematoxylin-eosin (HE). All the cases were evaluated according updated Sydney scoring system. The following items were evaluated separately: H. Pylori colonization, presence of an inflammatory component (the amount of neutrophilic infiltration), presence of a chronic inflammatory component (the amount of lymphoplasmacytic infiltration), mucosal atrophy, intestinal metaplasia, lymphoid follicles, dysplasia, and cell changes. All these items were scored to mild, moderate, severe.

\subsection{Statistical Analysis}

The data were entered and analyzed using the SPSS software (Version 25, SPSS Inc., Chicago, IL, USA). Chi-square test (Pearson value) was used. A p-value less than 0.05 was considered statistically significant.

\section{Results}

\subsection{Epidemiological data}

248 patient were included in this study. The main age was $49.45 \pm 17.41(\mathrm{n}=248$, range $=16-80$ years $)$, the male to female ratio was 0.7 , there was 107 males (43.1\%) and 141 Females (56.9\%).

When we investigated the presence of H. Pylori in our specimens, we found that 171 subjects are affected with a prevalence of $67 \%$.

\subsection{Gender}

The repartition according to gender showed that the prevalence of $H$. Pylori infection was higher in females comparing to males $(39.77 \%$ vs. $60.23 \%$ ) (Figure 1 ). Despite of that finding, there was no statistically significant relationship between gender and intensity of H. Pylori infection. $(\mathrm{p}=0.109)$.

\subsection{Age}

We performed an analysis to the prevalence of $H$. pylori by age groups, For this analysis, the age was divided into five subgroups as follows: $\leq 19$ years, 20 to 39 years, 40 to 59 years, 60 to 79 years, $>80$ years. The prevalence in each group was:

- Under 19: $3.87 \%$

- 20 to $39: 26.45 \%$

- $\quad 40$ to $59: 37.42 \%$

- 60 to $79: 30.32 \%$

- $\quad$ More than 80: $1.94 \%$

H. Pylori as well as chronic gastritis seems, to be present in all age groups and there was no statistical difference concerning the prediction to $H$. Pylori infection $(\mathrm{p}=0.07)$. Hence the factor age was not associated to an increased risk of infection. (Figure 2).

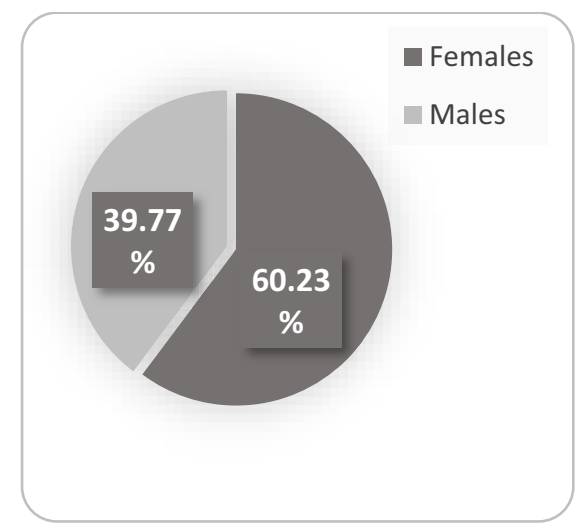

Fig.1. Distribution by gender of the prevalence of H. Pylori infection.

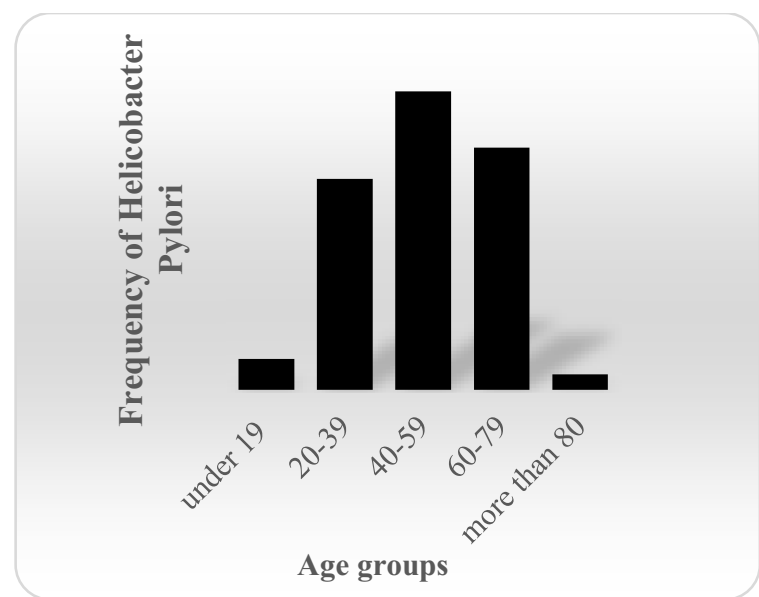

Fig.2. Distribution by age of the prevalence of H. pylori infection.

\subsection{Socioeconomic status}

In this study we divided patients into two groups according to their national health coverage system, and we investigated the frequency of $H$. Pylori infection in each group. The first group is patients who paid their tests or benefiting from a mandatory healthcare insurance (AMO). The other group

*Corresponding author: radia.elgui@uit.ac.ma 
comprises patients holding RAMED cards, which is a medical assistance plan for the neediest, allowing them to benefit from free public healthcare services. National health coverage system has been used as a proxy marker of socioeconomic status and an important determinant of $H$. Pylori prevalence in resource limited settings, we considered that patients that were benefiting from RAMED belong to low socioeconomic class and those who were benefiting from AMO or cash paying their medical cares are considered as middle to upper social class. The results as in figure 3 , revealed a statistically significant difference between the two groups since the first group was highly infected comparing to the second one $(71.93 \%$ Vs $28.07 \%)(p=0.04)$

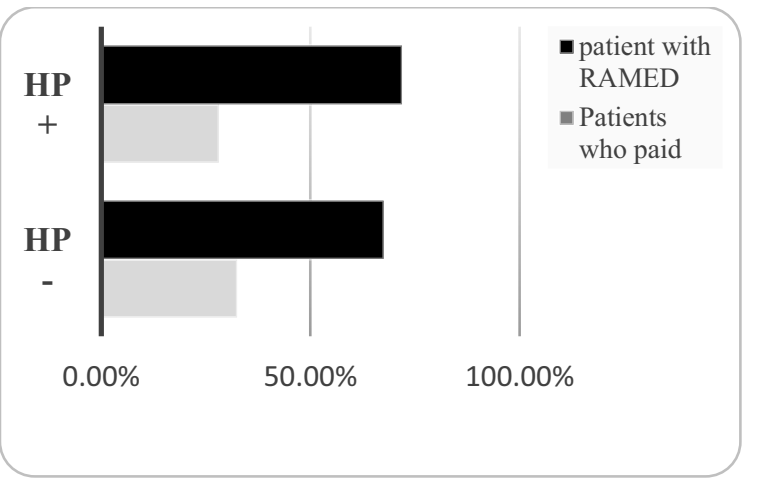

Fig.3. Distribution of $H$. Pylori according to national health coverage system

\subsection{Histological Features}

All 248 analyzed samples showed all histological chronic gastritis, otherwise $100 \%$ of our population is suffering from chronic gastritis, the prevalence of $H$. Pylori is $67 \%$.

\section{- Gastritis Intensity}

Among 248 subjects with chronic chronic gastritis, 80 $(32.26 \%)$ had mild intensity, $134(54.03 \%)$ moderate gastritis, and 34 (13.71\%) had severe gastritis.

We investigated the relation between density of Helicobacter pylori colonization in gastric mucosa and the gastric mucosal inflammation severity, we found out that, the severity of chronic gastritis was mostly reported as mild in people that are not infected or had mild $H$. pylori colonization respectively $61.04 \%$ and $52.27 \%$, As shown in figure 4 , the severity of the chronic gastritis tends to increase as long as the colonization of $H$. Pylori increases. There was a significant statistical relationship between the incidence of $H$. pylori infection and the severity of chronic gastritis $(\mathrm{p}<0.001)$.

- Gastritis Activity

In term of activity, the active chronic gastritis presented $74.58 \%$ of our samples, with different grade, $35.42 \%$ mild, $28.33 \%$ moderate, and $10.83 \%$ severe. The evaluation of the association between the CG activity and the $H$. Pylori infection revealed a significant difference.

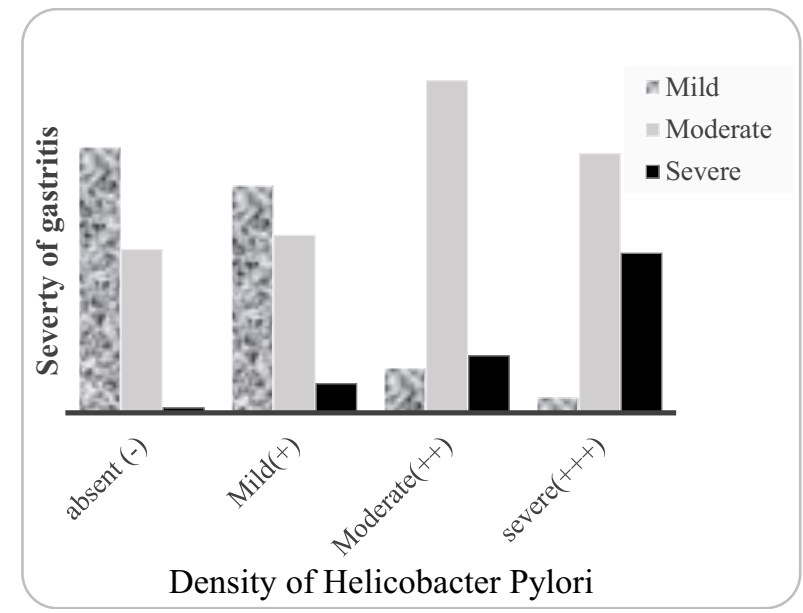

Fig.4. Association between severity of chronic gastritis and intensity of $H$. Pylori infection

As shown in Figure 5, we notice that patients with no Helicobacter Pylori infection have a highest rate of nonactive gastritis $(74.29 \%)$, in contrast those with severe H. Pylori colonization are mostly moderate to severely active; respectively $42.11 \%$ and $31.58 \%$. The level of activity increases by increased intensity of colonization there was a statistically significant association between the severity of $H$. pylori infection and the activity of chronic gastritis $\mathrm{P}<0.005)$.

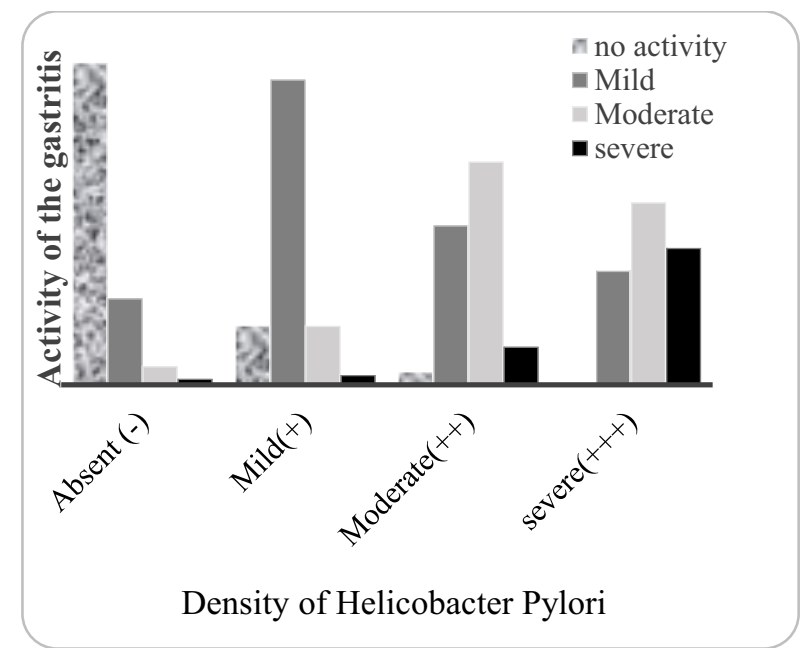

Fig.5. Association between the activity of the gastritis and intensity of H. Pylori infection

\section{- Glandular atrophy}

In our study glandular atrophy presented $10.37 \%(\mathrm{n}=$ 25 ), and $80 \%$ of those atrophic gastritis were associated with $H$. Pylori infection $(\mathrm{p}=0.004)$ and $12 \%$ related to autoimmune diseases (Pernicious anemia), in our study, there was no statistical correlation between Atrophic Gastritis and autoimmune diseases $(p=0.68)$. 


\section{- Intestinal metaplasia}

$10.53 \%$ gastric biopsies have shown Intestinal metaplasia $(\mathrm{n}=26)$, and $H$. Pylori was observed in 42 . $31 \%$ of cases $(n=11)$. Also, there was a statistically significant relationship between $H$. pylori infection and the intestinal metaplasia $(\mathrm{p}=0.001)$.

- Epithelial dysplasia

The dysplasia is detected in one case, in a 72 years old patient, accompanied with a metaplasia and it had very low density of H. Pylori.

\section{- Adenocarcinoma}

We also observed one case of gastric adenocarcinoma, of an 80 years old woman, the specimen had a low density of H. Pylori.

\section{- Lymphoid follicles}

Follicular gastritis (lymphoid follicles formations) are in $32 \%$ of cases $(\mathrm{n}=79)$, and they were more frequent in H. Pylori infected slides. $82 \%$ of subject carrying $H$. Pylori have shown presence of lymphoid follicles. There was a statistically significant association between the two last variables $(p<0.005)$.

\section{Discussion}

H. Pylori is known to colonize more than half of the world's population, but it prevalence varies depending on different contributing factors: socioeconomic status, living standards, ethnicity and geographical location [7]. The present study showed that the prevalence of $H$. Pylori infection is $67 \%$, similar result was observed in other Moroccan studies [8-10], and in other developing countries like Saudi Arabia 65.9, and India 63.5. But it's too quite low compared to neighboring countries like Tunisia (72\%) [11], Mauritania (76.4\%) [12], Algeria (78\%) [13].this number has been declining in developed and industrialized countries of the western world. Switzerland had the lowest reported $H$. Pylori prevalence $(18.9 \%), 35 \%$ in the United Kingdom, and $26 \%$ in Sweden. [11]

The prevalence of $H$. Pylori infection according to age is joining several studies that reported no significant difference of $H$. Pylori infection between different adult age groups [14-17], Nevertheless some studies reported that this prevalence increases with the age $[18,19]$. Zamani et al [20] explained this tendency by either birth cohort effect or accumulated risk of infection by age, as the risk of exposure increases with the age, and the infection occurs at a young age. Few studies were found about the H. Pylori infection age decreasing. [21,22] In terms of gender, our findings indicated that, there were no statistically significant differences in the prevalence of $H$. Pylori between men and women, both are exposed similarly to be infected by this bacteria and several studies sustain this result. [16,20,21]. Although there are controversial reports about this relation, Some authors reported a high prevalence of $\mathrm{H}$. pylori among females [18]. While other authors reported a high rate of
H. pylori infection among males [19]. Gender disparity in H. pylori infection is an intriguing topic.

Our results revealed that low socio-economic status is a predictive factor to helicobacter pylori infection, $71.93 \%$ of low-income patients were infected $(\mathrm{p}=0.04)$. Indeed, a large part of the population that goes to Provincial Kenitra Public Hospital are coming from rural and surrounding communes where the poverty rate $(9.7 \%)$ is quiet higher than the national average (4.8\%) [23]. Lifestyle and basic sanitation conditions favors person-to-person transmission of the microorganism. Hence low socio-economic status families with low income had a higher likelihood of carrying H. Pylori infection. More than few Moroccan and international studies confirmed this fact $[10,16,24,25]$.

CG has been histologically confirmed in all the specimens $(100 \%)$, which is a very high value. This prevalence can be explained by the fact that all the patient suffered from different clinical gastric disorders before getting gastro-oduodeno-fibroscopy.

H. Pylori is found in $67 \%$ of cases, the only similar study, which has been conducted in the same region by Attaf et al. in 2004 reported the same prevalence[26], nevertheless our finding is in the limit of a lot of recent Moroccan studies [8,27,28].

The degree of $H$. Pylori colonization was significantly associated to the severity of CG. In fact , The host Immune response to $H$. pylori invasion make up a complex network of inflammatory mediators (cytokines and chemotactic factors) that will have a direct cytopathic effect and will initiate the gastric inflammation that will result on lymphocyte and plasmocyte cell infiltration [29]. A meta-analysis conducted by Rokkas et al. in 2017, affirms, that $H$. pylori is a significant risk factor for GC and that its eradication is associated with a reduction in the incidence of GC [30]. Few studies rejected this notion of association [31].

H. Pylori induces epithelial cells to release chemokines that are chemotactic for neutrophils that infiltrates the lamina propria of the gastric mucosa and that defines the activity [32]. Neutrophil infiltration in the lamina propria is generally accepted to be the unanimous indicator of H. Pylori gastritis.

A study conducted by Ghasemi et al. in 2017, reported that there was a statistically significant correlation between the intensity of $H$. Pylori colonization and chronic gastritis [33], and this is in agreement with our findings and those of other researchers on the topic $[32,34]$, that confirm a coherent relationship. Though the literature shows some disagreeing results, both Choudhary et al. and Park et al. are attesting that $H$. Pylori infection doesn't have any effect on activity of chronic gastritis, which can be related to genetic differences, nutritional habits, and environmental factors between the studied populations [31,35].

The development of $\mathrm{CG}$ constitutes a complex progression of events, characterized mainly by interactions between H. Pylori infection, host factors

${ }^{*}$ Corresponding author: radia.elgui@uit.ac.ma 
and environmental influences, In the gastric mucosa, $\mathrm{H}$. pylori initiates a cascade events, which may lead to chronic atrophic Gastritis (CAG) and then to Intestinal Metaplasia (IM), Dysplasia, and finally carcinoma. [1] The response of surface epithelium to $H$. Pylori infection is mucus depletion, desquamation of the epithelium and regenerative change. Loss of the glands in the gastric mucosa is known as atrophy. Corporal atrophy induced by free oxygen radicals released from neutrophils is closely linked to the acid secretion suppression, and thus a reduction of gastric secretory function [36]

We reported $10.37 \%$ of atrophic gastritis and $10.53 \%$ of Intestinal Metaplasia. The prevalence of gastric IM and atrophy in the general population is known to vary around the globe, mostly depending on $H$. pylori status $[33,36,37]$. Our result joint those already found in Morocco. Bounder et al reported in his study $11.7 \%$ of AG and $7.4 \%$ of IM.

We found that $80 \%$ of our atrophic gastritis form were related to Helicobacter Pylori infection, such findings are supported by a substantial number of studies $[34,38,39]$. Asaka et al. reported the same prevalence (the prevalence of atrophic gastritis in $\mathrm{H}$. pylori infection was $82.9 \%$ ) [40]. In the another study, Marshall stated that H. Pylori is the origin of $80 \%$ of AG and the study of Attaf et al. was conducted in the same region affirmed the schema[26] .Statistically, the atrophic gastritis is a potential risk factor for cancer than the pure H. pylori infection. The likelihood of gastric cancer is much higher with the progression of the $H$. pylori gastritis from a non-atrophic form to an atrophic form [1].

Intestinal metaplasia is considered to be an advanced stage of atrophy. It is defined as replacement of gastric epithelial cells by cells of intestinal morphology. $10.53 \%$ IM was reported and mostly belong to $60-79$ years old age group (57\%). A statistically significant relation between IM and $H$. Pylori, $42.31 \%$ with IM were infected.

There is a wide variation in the prevalence of gastric IM depending on the different methods used in studies and particularly the prevalence of $H$. Pylori infection in the region.

A Chinese study conducted on 435 subjects, revealed that persistent $H$. Pylori infection, age $>45$ years, were significantly associated with IM progression [41]. Asaka et al. studied the relation of H. Pylori with IM. They found the rates of IM were $43.1 \%$ in the infected group and $6.2 \%$ in non-infected groups [40].

Rokkas et al. suggest That H. Pylori eradication is a primary preventive strategy in subject with non-atrophic or atrophic gastritis, In contrast, H. Pylori eradication for patients who have already developed advanced preneoplastic lesions such IM or Dysplasia, does not prevent CG development. All this supports the notion that IM represents " the point of no return " [30]. Subsequent studies have confirmed a close association between IM progression and gastric carcinoma, with odds ratios of progression to neoplasia ranging from 5 to 29 depending on the studied population. IM is now considered a true preneoplastic lesion of the stomach [42].

One case of dysplasia and one case of adenocarcinoma were found in our study, both subjects are more than 70 years old and both had low H. Pylori colonization. Kuiper had demonstrated that after 30 years, of the $H$. Pylori lesion progression, there is a chance of $1 \%$ to develop gastric cancer [43].

H. Pylori infection may provide the proper environment for atrophic gastritis and intestinal metaplasia to occur. Paradoxically at the final stage of the disease, intestinal metaplasia and dysplasia are not a suitable environment for H. Pylori growth. So we generally notice dramatic reduction or even disappearance of the organism [44].

There is conflicting data about the rapidity of transition from the atrophic form to metaplasic form and then from the dysplasia to the neoplastic which makes it difficult to elaborate surveillance recommendations for subjects having pre-cancerous lesions.

Lymphoid follicles were found in $32 \%$ of cases, which is relatively lower than the results of Ghesemi et al. that found $46.11 \%$ of lymphoid follicles in their studies [33]. However, Daré et al. reported 19.84\%.

The careful examination of specimens revealed that in the gastric mucosa was present in $80 \%$ of patients with $H$ pylori infection, and that there is a statistically significant difference between $H$. Pylori infection and lymphoid follicles, what suggest that H. Pylori is an important risk factor for the formation of lymphoid follicles.

This is agreement with the findings of other studies on the topic $[32,45]$. In fact The significant association of H. Pylori and lymphoid follicles in this study indicates that the bacterium may induce local humoral and T-cell response in the gastric mucosa. H. Pylori infection triggers the development of MALT tissue, which can act as a nest for the potential development of a lymphoma.

\section{Conclusion}

In the model of gastric carcinogenesis, $H$. pylori plays a pivotal role in the initiation and the progression of chronic active gastritis. The infection progress over years through the sequential stages of atrophic gastritis, Intestinal Metaplasia, dysplasia and to gastric adenocarcinoma. Therefore, it seems that an early screening and an early eradicating at an early stages of the infection is the most viable strategy to prevent the long term lesions of the chronic gastritis, it should be considered as a primordial health goal.

\section{References}

1. BJ. Marshall, JR. Warren. Unidentified curved bacilli in the stomach of patients with gastritis and peptic ulceration. Lancet Lond. Engl. 1(8390), 1311-1315 (1984). 
2. B. Zabala Torrres, Y. Lucero, AJ. Lagomarcino et al. Review: Prevalence and dynamics of Helicobacter pylori infection during childhood. Helicobacter. 22(5), e12399 (2017).

3. Guillemot G. Étude Hélirun, épidémiologie de l'infection à Hélicobacter pylori et de la résistance à la clarithromycine à la Réunion en 2014 : étude chez 200 patients adultes ayant une indication d'endoscopie digestive supérieure au CHU de Saint Pierre [Internet]. (2015).

4. Egan BJ, O'Morain CA. A historical perspective of Helicobacter gastroduodenitis and its complications. Best Pract. Res. Clin. Gastroenterol. 21(2), 335-346 (2007).

5. Mathers CD, Loncar D. Projections of Global Mortality and Burden of Disease from 2002 to 2030. PLOS Med. 3(11), e442 (2006).

6. Correa P, Piazuelo MB. The gastric precancerous cascade. J. Dig. Dis. 13(1), 2-9 (2012).

7. de Korwin J-D. [Helicobacter pylori 30 years after: What's new?]. Rev. Med. Interne. 35(9), 561-564 (2014).

8. El Khadir M, Alaoui Boukhris S, Benajah D-A, et al. Helicobacter pylori CagA EPIYA-C motifs and gastric diseases in Moroccan patients. Infect. Genet. Evol. 66, 120-129 (2018).

9. Joutei HAH, Hilali A, Fechtali T, Rhallabi N, Benomar $\mathrm{H}$. Helicobacter pylori infection in 755 patients with digestive complaints: Pasteur Institute, Morocco, 19982007. East. Mediterr. Health J. 16(7), 778-782 (2010).

10. Sokpon M, Salihoun M, Lahlou L, Acharki M, Razine R, Kabbaj N. Facteurs prédictifs de l'infection à Helicobacter pylori (Hp) au cours de la gastrite chronique: à propos d'une étude marocaine. J. Afr. Hépato-Gastroentérologie. 10 (2016).

11. Global Prevalence of Helicobacter pylori Infection: Systematic Review and Meta-Analysis. Gastroenterology. 153(2), 420-429 (2017).

12. Bouh KO, Jidou MMT, El Bara A. Séroprévalence de l'infection à Helicobacter Pylori dans une population mauritanienne asymptomatique. $J$. Afr. HépatoGastroentérologie. 9(1), 18-21 (2015).

13. Bachir M, Allem R, Benejat L, et al. Molecular detection of mutations involved in Helicobacter pylori antibiotic resistance in Algeria. J. Antimicrob. Chemother. 73(8), 2034-2038 (2018).

14. Adlekha S, Chadha T, Krishnan P, Sumangala B. Prevalence of helicobacter pylori infection among patients undergoing upper gastrointestinal endoscopy in a medical college hospital in kerala, India. Ann. Med. Health Sci. Res. 3(4), 559-563 (2013).

15. Eusebi LH, Zagari RM, Bazzoli F. Epidemiology of Helicobacter pylori Infection. Helicobacter. 19, 1-5 (2014).

16. Alvarado-Esquivel C. Seroepidemiology of helicobacter pylori infection in pregnant women in rural durango, Mexico. Int. J. Biomed. Sci. IJBS. 9(4), 224-229 (2013).

17. Porras C, Nodora J, Sexton R, et al. Epidemiology of Helicobacter pylori infection in six Latin American countries (SWOG Trial S0701). Cancer Causes Control CCC. 24(2), 209-215 (2013).

18. Khoder G, Muhammad JS, Mahmoud I, Soliman SSM, Burucoa C. Prevalence of Helicobacter pylori and Its Associated Factors among Healthy Asymptomatic Residents in the United Arab Emirates. Pathogens. 8(2), 44 (2019).

19. Bruden DL, Bruce MG, Miernyk KM, et al. Diagnostic accuracy of tests for Helicobacter pylori in an Alaska Native population. World J. Gastroenterol. 17(42), 4682-4688 (2011).

20. Zamani M, Ebrahimtabar F, Zamani V, et al. Systematic review with meta-analysis: the worldwide prevalence of Helicobacter pylori infection. Aliment. Pharmacol. Ther. 47(7), 868-876 (2018).

21. Ashtari S, Pourhoseingholi MA, Molaei M, Taslimi H, Zali MR. The prevalence of Helicobacter pylori is decreasing in Iranian patients. Gastroenterol. Hepatol. Bed Bench. 8(1), 23-29 (2015).

22. Chen S, Ying L, Kong $\mathrm{M}$, Zhang $\mathrm{Y}$, Li Y. The Prevalence of Helicobacter pylori Infection Decreases with Older Age in Atrophic Gastritis. Gastroenterol. Res. Pract. 2013, e494783 (2013).

23. Royaume du Maroc: Haut Commissariat Du Plan. Pauvreté | Site de la Direction Provinciale de Kénitra (2014)

24. Benajah DA, Lahbabi M, Alaoui S, et al. Prevalence of Helicobacter pylori and its recurrence after successful eradication in a developing nation (Morocco). Clin. Res. Hepatol. Gastroenterol. 37(5), 519-526 (2013).

25. Awuku YA, Simpong DL, Alhassan IK, Tuoyire DA, Afaa T, Adu P. Prevalence of helicobacter pylori infection among children living in a rural setting in Sub-Saharan Africa. BMC Public Health. 17(1), 1-6 (2017).

26. Attaf N, Cherkaoui N, Choulli MK, Ghazali L, Mokhtari A, Soulaymani A. Profil épidémiologique de l'infection à Hélicobacter pylori dans la région du Gharb-Chrarda-Beni Hssen. 4, 11 (2004). 
27. Khadir ME, Boukhris SA, Benajah D-A, et al. VacA and CagA Status as Biomarker of Two Opposite End Outcomes of Helicobacter pylori Infection (Gastric Cancer and Duodenal Ulcer) in a Moroccan Population. PLOS ONE. 12(1), e0170616 (2017).

28. Cherif S, Rais H, Hakmaoui A, Sellami S, Elantri S, Amine A. Linking Helicobacter pylori with gallbladder and biliary tract cancer in Moroccan population using clinical and pathological profiles. Bioinformation. 15(10), 735-743 (2019).

29. Carl-McGrath S, Ebert M, Röcken C. Gastric adenocarcinoma: Epidemiology, pathology and pathogenesis. Cancer Ther. 5, 877-894 (2007).

30. Rokkas T, Rokka A, Portincasa P. A systematic review and meta-analysis of the role of Helicobacter pylori eradication in preventing gastric cancer. Ann. Gastroenterol. 30(4), 414-423 (2017).

31. Choudhary CK C, Uk B, A A, Rs G. Correlation of H. pylori density with grading of chronic gastritis. Indian J. Pathol. Microbiol. 44(3), 325-328 (2001).

32. Yakoob MY, Hussainy AS. Chronic gastritis and Helicobacter pylori: a histopathological study of gastric mucosal biopsies. 20, 4 (2010).

33. Ghasemi Basir HR, Ghobakhlou M, Akbari P, Dehghan A, Seif Rabiei MA. Correlation between the Intensity of Helicobacter pylori Colonization and Severity of Gastritis. Gastroenterol. Res. Pract. (2017).

34. Turkay C, Erbayrak M, Bavbek N, Yenidunya S, Eraslan E, Kasapoglu B. Helicobacter pylori and histopathological findings in patients with dyspepsia. Turk. J. Gastroenterol. 22(2), 122-127 (2011).

35. Park J, Kim MK, Park SM. Influence of Helicobacter pylori Colonization on Histological Grading of Chronic Gastritis in Korean Patients with Peptic Ulcer. Korean J. Intern. Med. 10(2), 125-129 (1995).

36. de Vries, A.C., Haringsma, J. and Kuipers, E.J. The detection, surveillance and treatment of premalignant gastric lesions related to Helicobacter pylori infection. Helicobacter, 12, 1-15(2007).

37. Spence AD, Cardwell CR, McMenamin ÚC, et al. Adenocarcinoma risk in gastric atrophy and intestinal metaplasia: a systematic review. BMC Gastroenterol. 17(1), 1-9 (2017).

38. Kwon YJ, Kim N, Baek SM, et al. The prevalence of histologic atrophy and intestinal metaplasia in the corpus has decreased over 15 years in females in the Korean population. Helicobacter. 24(3), e12579 (2019).

39. Song JH, Kim YS, Heo NJ, et al. High Salt Intake Is Associated with Atrophic Gastritis with Intestinal
Metaplasia. Cancer Epidemiol. Prev. Biomark. 26(7), 1133-1138 (2017)

40. Asaka M, Sugiyama T, Nobuta A, Kato M, Takeda H, Graham DY. Atrophic gastritis and intestinal metaplasia in Japan: results of a large multicenter study. Helicobacter. 6(4), 294-299 (2001).

41. Leung WK, Lin S-R, Ching JYL, et al. Factors predicting progression of gastric intestinal metaplasia: results of a randomised trial on Helicobacter pylori eradication. Gut. 53(9), 1244-1249 (2004).

42. Giraud AS. Metaplasia as a Premalignant Pathology in the Stomach. Gastroenterology. 132(5), 2053-2056 (2007).

43. Kuipers EJ. Review article: exploring the link between Helicobacter pylori and gastric cancer. Aliment. Pharmacol. Ther. 13(s1), 3-11 (1999).

44. Zhang C, Yamada N, Wu Y-L, Wen M, Matsuhisa T, Matsukura N. Helicobacter pylori infection, glandular atrophy and intestinal metaplasia in superficial gastritis, gastric erosion, erosive gastritis, gastric ulcer and early gastric cancer. World J. Gastroenterol. WJG. 11(6), 791-796 (2005).

45. Genta RM, Graham DY. Comparison of biopsy sites for the histopathologic diagnosis of Helicobacter pylori: a topographic study of H. pylori density and distribution. Gastrointest. Endosc. 40(3), 342-345 (1994). 